\title{
Differential Chaos Shift Keying: A Robust Modulation Scheme for Power-Line Communications
}

\author{
Georges Kaddoum*, Member, IEEE, and Navid Tadayon, Member, IEEE
}

\begin{abstract}
The past few years have witnessed a tremendous development in power-line communications (PLC) for the realization of smart grids. Since power lines were not originally intended for conveying high frequency signals, any communication over these lines would be exposed to severe adversarial factors, such as interference, impulsive and phase noise. This elucidates the importance of employing robust modulation techniques and motivates research in this direction. Indeed, the aim of the paper is to propose a differential chaos shift keying (DCSK) modulation scheme as a potential candidate for smart grid communication networks. This DCSK class of non-coherent modulation is very robust against linear and non-linear channel distortions. More importantly, the demodulation process can be carried out without any channel estimator at the receiver side. In this work, we analyse the bit error rate (BER) performance of DCSK over multipath PLC channels in which phase, background and impulsive noise are present. A simulator is developed to verify the performance of the proposed DCSK against direct sequence code division multiple access (DS-CDMA) and direct sequence differential phase shift keying (DS-DPSK). The results presented in this work prove the advantages of this low-cost noncoherent modulation technique for PLC systems over its rivals.
\end{abstract}

Index Terms-Non-coherent chaos-based communication system, PLC, Impulsive noise, Performance analysis.

\section{INTRODUCTION}

Despite the fact that power-line communications (PLC) have been around for more than a century, it has only recently received particular attention due to the emergence of interesting novel applications. A paradigm known as smart grids drives a fast track adoption of the PLC technology. This recent interest in smart grids stems from the imminent increase in electricity needs of our societies. To cater to such burgeoning demands, one shall realize that power generation/distribution can no longer be fulfilled locally and in a static manner. In fact, our existing power grid systems are operating way below their optimal points and are inert for the lack of coordination, agility, and feedback from fields. Considering the scale of power grids in a country like United States, where more than 9000 power generating units are pumping almost one million megawatts of electricity into more than 300, 000 miles of transmission lines, adding intelligence and autonomy can hugely improve the generation, distribution,

G. Kaddoum and N. Tadayon are with University of Québec, ÉTS, LaCIME Laboratory, 1100 Notre-Dame west, H3C 1K3, Montreal, Canada (e-mail: georges.kaddoum@etsmtl.ca, tadayon@emt.inrs.ca)

* This work has been supported by the NSERC discovery grant $435243-$ 2013. and utilization efficiency [1]. Moreover, such coordination can effectively reduce maintenance and restoration costs and delays, handle peak demands and improve security measures. An economically justifiable and smooth solution is to send data and electricity over the power line (PL) commodities, concurrently. Further to the aforementioned remote sensing role, PLC can be a ubiquitous platform capable of offering high data rate access to information in most populated cities as well as remote areas on a plug-and-play basis [1]. In other words, PLC is a cheap alternative to both the digital subscriber line (DSL) and the optical networking and wireless regional area networks (WRANs). Notwithstanding its advantages, PLC faces several disadvantages if utilized in its current unaltered form. For the most part, power transmission medium is not ideal to carry signals with frequencies much higher than $50-60 \mathrm{~Hz}$. In fact, experimental results unveiled attenuation as high as $10-30 \mathrm{~dB}$ for data transmission over low voltage lines and $100 \mathrm{~dB}$ for transmission over medium and high voltage lines. Additionally, transformers can easily filter out the carrier signal, altogether. Beyond all these, interference induced from outside sources into PLs, multipath corruptions and the back-propagation of noise into the wires from loads such as switching devices and home appliances can superimpose and render the received signal completely undecodable [2]. It is important to note that the coherence time of different channel gains in a PLC system is high [2].

To tackle these shortcomings and obstacles, separate standardization working groups, such as IEEE P. 1901 and European telecommunication standard institute (ETSI), are currently working toward developing robust modulation schemes for PLC. Among many proposals, wideband modulation schemes such as orthogonal frequency division multiplexing (OFDM) and code division multiple access (CDMA) have gained prominence and been considered for possible adoption into futuristic PLC architecture [3].

On the other hand, three types of noise can severely hinder communication over PLs: background noise [4], phase noise and impulsive noise. Many communication systems assume that the background noise in PLC is an additive white Gaussian noise (AWGN). This is usually done to derive the analytical closed-form expression bounds of several performance metrics. Indeed, it has been experimentally verified in [5]-[7] that this latter follows the Nakagami-m distribution. The phase and impulsive noise have random but periodic natures and occur in short bursts. Impulse noise has much wider power spectral density (PSD) than the background and phase noise [5]-[7]. 
To alleviate the impairments caused by these three noise types, chaotic-based modulation is proposed in this paper. This is in motivation by the fact that chaotic signals can be generated using off-the-shelf electronic devices. Moreover, due to their high sensitivity to initial conditions, chaotic maps can generate infinite number of signals with extremely low cross-correlation levels, at least in theory. Owing to their favorable wideband characteristics, chaotic signals have proved to be one of the native candidates for multi-user spread-spectrum modulation schemes [8]-[10].

Moreover, several studies have addressed multi-user interference and the peak-to-average power ratio (PAPR) mitigation using chaos-based sequences proving that the latter outperforms exploitation of Gold sequences in multi-user spread-spectrum communication systems [11]. Among various existing chaotic-based schemes, differential chaos shift keying (DCSK) gained recent popularity [10] due to its needlessness to either synchronization or channel state information (CSI) as well as its simple non-coherent detection.

Moreover, despite the fact that detection in DCSK and differential phase shift keying (DPSK) is done non-coherently where both are needless of CSI at the receiver side [12], [13], DCSK is more resistant against multipath fading and nonlinear distortions. This renders it an attractive alternative for ultra wide band (UWB) systems [9], [10], [13].

Contributions: In this paper, we introduce DCSK as a robust and inexpensive modulation scheme for power-line communications. The underpinning reason for such choice is implementation simplicity and its robustness against linear and non-linear channel distortions. To prove this claim, DCSK performance over PL channels with multiple echoes and in the presence of background, impulsive, and phase noise is evaluated through the derivation of closed-form analytical expression of the bit error rate (BER) and developing a systemlevel simulator. For validation purpose, the performance of DCSK is compared with DS-DPSK and DS-CDMA systems. To the best of our knowledge, this study is the first to analyze the performance of DCSK in PLC systems. In fact, the choice of DCSK system is because of its designation as a prominent benchmark for transmit reference non-coherent modulation class.

\section{DCSK SYSTEM MODEL AND DESCRIPTION FOR PLC Channels}

As shown in Fig. 1, each bit $b_{i}=\{-1,+1\}$ in the DCSK modulator is represented by a chaotic signal that is comprised of two parts; the first part is allocated to the reference symbol, and the second part to the data-carrying symbol. If +1 is transmitted, the data-carrying sequence is equal to the reference sequence, and if -1 is transmitted, an inverted version of the reference sequence is used as the data-carrying sequence. In the present system, $T_{\mathrm{DCSK}}=2 T_{b}=2 \beta T_{c}$ is the length of DCSK frame interval for each bit, where $T_{c}$ is the chip-time, $\beta$ is an integer referring to the reference length, and $T_{b}$ is the bit duration. The bandwidth spreading factor in DCSK is defined as the ratio of the bit period $T_{b}$ to the chip period $T_{c}$ which is equal to $\beta$. During the $i^{\text {th }}$ bit interval, the

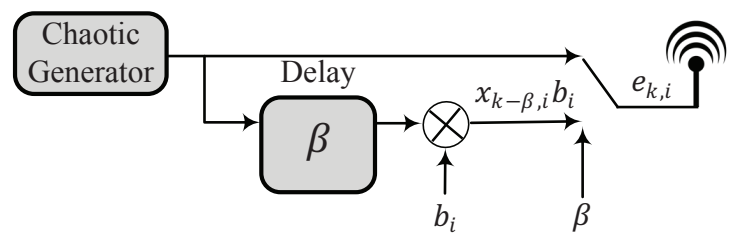

(a) DCSK transmitter.

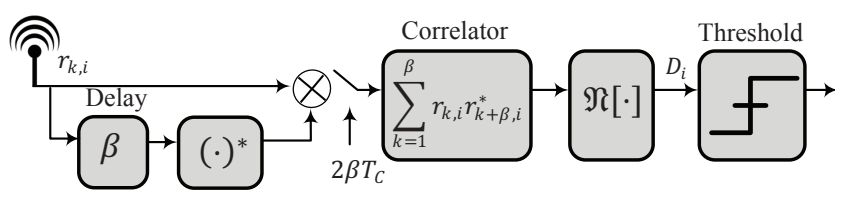

(b) DCSK receiver.

Fig. 1: A diagrammatic representation of DCSK transmitter/receiver.

discrete form of the baseband signal sequence at the output of the transmitter, denoted by $e_{n, i}$, is given by

$$
e_{n, i}=\left\{\begin{array}{ll}
x_{n, i}, & 1<n \leq \beta \\
b_{i} x_{n-\beta, i}, & \beta<n \leq 2 \beta
\end{array},\right.
$$

where $x_{n, i}$ is the chaotic sequence used as the reference signal and $x_{n-\beta, i}$ is its delayed version. As depicted in Fig. 2, the channel model considered in this paper is the echo model developed for the PL channel in [14] whose impulse response is denoted by

$$
h(t)=\sum_{l=1}^{L} \alpha_{l} \delta\left(t-\tau_{l}\right),
$$

where $\delta(t)$ is the impulse function, $\alpha_{l}, \tau_{l}$ are the gain and delay of the $l^{\text {th }}$ path, respectively, and $L$ is the number of existing paths. When propagating through the PL, the DCSK signal is corrupted with the impulsive and background noise. Hence, the total additive noise can be modeled as

$$
Y_{n}=w_{n}+I_{n},
$$

where $Y_{n}$ is the total noise, $w_{n}$ is a complex AWGN with variance $\sigma_{w}=N_{0}$, and $I_{n}$ is the impulsive complex AWGN noise with variance $\sigma_{I}=N_{I}$. All the components in (3) are mutually independent. In this paper, we consider the twomixture Gaussian model [3], [15] to represent the PLC channel impulsive noise that is characterized as follows

$$
I_{n}=B_{n} g_{n},
$$

where $g_{n}$ is a zero mean white Gaussian process and $B_{n}$ is a Poisson process whose probability mass function (PMF) is given by [16],

$$
P\left(B_{n}=k\right)=\frac{e^{-\lambda t}(\lambda t)^{k}}{k !}, k=0,1,2, \ldots
$$

This equation describes the probability that $k$ impulses of noise arrive within $t$ seconds and $\lambda$ represents the average occurrence rate of the impulse noise. The multiplicative model in (4) describes a process with random amplitude $g_{n}$ that affects each transmitted symbol independently and randomly according to the probability distribution of $B_{n}$. In our model, 


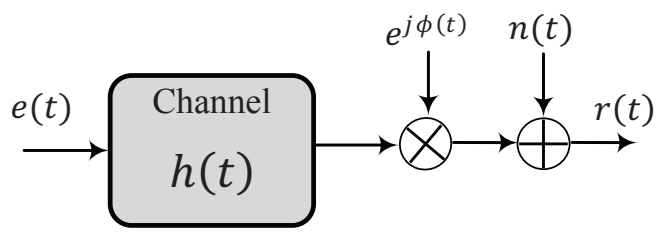

Fig. 2: Power line echo channel in the presence of background, impulsive and phase noise.

we take into account the effect of phase noise $\phi_{n}$ on the performance of PLC [2] by having it modeled as a WinnerLèvy process (or random walk), which is formulated, in discrete form, according to $\phi_{n}=2 \pi \sum_{t=0}^{n} \mu(u) \mathrm{d} u$ [17]. In this equation, $\mu(u)$ is a zero-mean Gaussian noise with variance $\sigma_{\mu}=N_{0, \mu}$. Putting all above factors altogether, the received signal is abstractly written as

$$
r_{n, i}=\sum_{l=1}^{L} \alpha_{l} e_{n-\tau_{l}, i} \mathrm{e}^{j \phi_{n, i}}+Y_{n} .
$$

To recover the data at the receiver, the received signal is first correlated with the complex conjugate of its delayed version. Then, the real part of this correlated output is extracted and compared to a threshold. This correlation process is illustrated in Fig. 1b. In this analysis, it is assumed that the largest delay $\tau_{L}$ is much shorter than the chaotic sequence duration, that is $0<\tau_{L} \ll \beta$, which results in negligible intersymbol interference (ISI) [9], [13]. It can be verified that above condition holds for PLC channels [14]. The decision variable at the output of the correlator, on the $i^{\text {th }}$ bit, would be equal to

$$
\begin{aligned}
D_{i}=\Re\left[T_{c} \sum_{n=1}^{\beta}\right. & \left(\sum_{l=1}^{L} \alpha_{l} b_{i} x_{n-\tau_{l}, i} \mathrm{e}^{j \phi_{n, i}}+Y_{n}\right) \times \\
& \left.\left(\sum_{l=1}^{L} \alpha_{l} x_{n-\tau_{l}, i} \mathrm{e}^{j \phi_{n, i}}+Y_{n+\beta}\right)^{*}\right],
\end{aligned}
$$

where $\Re$ and ${ }^{*}$ are the real-part and complex conjugate operators. Using the approximation $\sum_{n=1}^{\beta} x_{n-\tau_{l}} x_{n-\tau_{m}} \approx$ $0, l \neq m$, which is valid for large reference length $\beta$, the variable $D_{i}$ may be further simplified as

$$
\begin{aligned}
D_{i}=T_{c} \sum_{n=1}^{\beta} \Re & {[\underbrace{\sum_{l=1}^{L}\left|\alpha_{l}^{2}\right| b_{i} x_{n-\tau_{l}, i}^{2}}_{\mathrm{C}_{1}}+\underbrace{\sum_{l=1}^{L} \alpha_{l} b_{i} x_{n-\tau_{l}, i} \mathrm{e}^{j \phi_{n, i}} Y_{n+\beta}^{*}}_{\mathrm{C}_{2}}} \\
& +\underbrace{\sum_{l=1}^{L} \alpha_{l} x_{n-\tau_{l}, i} \mathrm{e}^{-j \phi_{n, i}} Y_{n}}_{\mathrm{C}_{3}}+\underbrace{Y_{n} Y_{n+\beta}^{*}}_{\mathrm{C}_{4}}] .
\end{aligned}
$$

As seen in (8), through the multiplication of the received signal with its complex conjugate replicate at the receiver, the first term $\mathrm{C}_{1}$ becomes independent of the phase noise making this modulation suitable for PLC applications. To generate chaotic sequences at the transmitter, the second-order Chebyshev polynomial function (CPF) $x_{n+1}=1-2 x_{n}^{2}$ is employed [18]. The variance of the normalized chaotic map with zero-mean is equal to one, i.e. $\operatorname{Var}[x]=\mathbb{E}\left[x^{2}\right]=1$, where $\mathbb{E}[\cdot]$ denotes the expectation operator. For mathematical tractability, $T_{c}=1$ throughout this article.

\section{Performance Analysis of DCSK over PlC ChanNels}

According to the above analysis, the net BER of the DCSK system over multipath PL channels, when impulsive and AWGN noise exist, can be written as $P_{b}^{\mathrm{T}}=p P_{b}^{\mathrm{I}}+(1-p) P_{b}$, where $p=\lambda T_{n}$ is the probability that a bit transmitted with DCSK is affected by the impulsive noise. Here, the quantities $P_{b}^{\mathrm{T}}, P_{b}^{\mathrm{I}}$, and $P_{b}$ are the total BER, the BER in the presence of impulsive noise, and the BER without impulsive noise, respectively. Also, $\lambda$ is the arrival rate and $T_{n}$ is the duration of the impulsive noise. To derive an expression for $P_{b}^{\mathrm{I}}$, the mean and the variance of the decision variable given in (8) are needed. One should note that the signal components of the decision variable in (8) are independent as the channel gains, the chaotic sequences and the noise components are all statistically independent. In addition, the chaotic sequence is independent of the noise components. As such, for the $i^{\text {th }}$ bit, the mean of the decision variable is the expectation of the useful signal, namely its first component $\mathrm{C}_{1}$. Thus,

$$
\mathbb{E}\left[D_{i}\right]=\frac{1}{2} b_{i} \sum_{l=1}^{L}\left|\alpha_{l}^{2}\right| E_{b},
$$

where $E_{b}=2 \sum_{n=1}^{\beta} \mathbb{E}\left[x_{n, i}^{2}\right]$ is the energy of the transmitted DCSK bit. It can be readily seen from (9) that the recovered useful energy is independent from the phase noise $\phi(t)$ which makes this modulation robust to phase noise interference. To derive the variance of $D_{i}$, we take advantage of the fact that, for statistically independent terms in (8), the variance of their sum is equal to the sum of individual term's variances. Having this in mind, since $Y_{n}$ and the chaotic signal in (8) are uncorrelated, the variance of $\mathrm{C}_{2}$ would be equal to ${ }^{1}$

$$
\begin{aligned}
\operatorname{Var}\left[\mathrm{C}_{2}\right] & =\frac{1}{2}\left(\frac{N_{0}}{2}+\frac{N_{I}}{2}\right) \sum_{l=1}^{L}\left|\alpha_{l}^{2}\right| E_{b} \mathbb{E}\left[\cos \left(\phi_{i}\right)^{2}\right] \\
& =\frac{1}{4}\left(\frac{N_{0}}{2}+\frac{N_{I}}{2}\right) \sum_{l=1}^{L}\left|\alpha_{l}^{2}\right| E_{b},
\end{aligned}
$$

where the simplification is due to the fact $\mathbb{E}\left[\cos \left(\phi_{i}\right)^{2}\right]=0.5$. By analogy, the third component in (8) has the same variance

$$
\operatorname{Var}\left[\mathrm{C}_{3}\right]=\frac{1}{4}\left(\frac{N_{0}}{2}+\frac{N_{I}}{2}\right) \sum_{l=1}^{L}\left|\alpha_{l}^{2}\right| E_{b} .
$$

Eventually, $\operatorname{Var}\left[\mathrm{C}_{4}\right]$ is obtained as,

$$
\operatorname{Var}\left[\mathrm{C}_{4}\right]=\beta\left(\frac{N_{0}^{2}}{2}+\frac{N_{I}^{2}}{2}\right) .
$$

${ }^{1}$ Note that $V\left[C_{2}\right], V\left[C_{3}\right]$, and $V\left[C_{4}\right]$ are the variances of real parts of the complex random variables $C_{2}, C_{3}$, and $C_{4}$. 
Summing up the terms in (10), (11), and (12) results in the variance of $D_{i}$ as

$$
\operatorname{Var}\left[D_{i}\right]=\frac{N_{0}+N_{I}}{4} \sum_{l=1}^{L}\left|\alpha_{l}^{2}\right| E_{b}+\frac{N_{0}^{2}+N_{I}^{2}}{2} \beta .
$$

Since bit energies (or chaotic chips) are deterministic variables, by virtue of the central limit theorem, the decision variable at the output of the correlator can be approximated by a Gaussian distribution. Knowing the fact that $\mathrm{BER}=0.5\left(\operatorname{Pr}\left(D_{i}<0 \mid b_{i}=+1\right)+\operatorname{Pr}\left(D_{i}>0 \mid b_{i}=-1\right)\right)$, the expression for BER becomes

$$
P_{b}^{\mathrm{I}}=\frac{1}{2} \operatorname{erfc}\left(\left(\frac{2 \operatorname{Var}\left[D_{i}\right]}{\mathbb{E}\left[D_{i}\right]^{2}}\right)^{-0.5}\right),
$$

where $\operatorname{erfc}(x)$ is the complementary error function defined as $\operatorname{erfc}(x) \equiv 2 / \sqrt{\pi} \int_{x}^{\infty} \exp \left(-\mu^{2}\right) \mathrm{d} \mu$. For large reference length $\beta$, the transmitted bit energy $E_{b}$ can be considered constant for all transmitted bits. On the other hand, when the reference length is short, the energy variation must be taken into account [18]. We adopt a constant bit energy for large values of $\beta$. By plugging (9) and (13) into (14), the BER for the DCSK over multipath channels, and in the presence of impulsive and AWGN noise, boils down to

$$
P_{b}^{\mathrm{I}}=\frac{1}{2} \operatorname{erfc}\left(\left(\frac{2\left(N_{0}+N_{I}\right)}{\sum_{l=1}^{L}\left|\alpha_{l}^{2}\right| E_{b}}+\frac{4 \beta\left(N_{0}^{2}+N_{I}^{2}\right)}{\left(\sum_{l=1}^{L}\left|\alpha_{l}^{2}\right| E_{b}\right)^{2}}\right)^{-0.5}\right) .
$$

By substituting $N_{I}=0$, the BER at the absence of impulsive noise, i.e. $P_{b}$, is attained

$$
P_{b}=\frac{1}{2} \operatorname{erfc}\left(\left(\frac{2 N_{0}}{\sum_{l=1}^{L}\left|\alpha_{l}^{2}\right| E_{b}}+\frac{4 \beta N_{0}^{2}}{\left(\sum_{l=1}^{L}\left|\alpha_{l}^{2}\right| E_{b}\right)^{2}}\right)^{-0.5}\right) .
$$

Finally, by plugging (15) and (16) into $P_{b}^{\mathrm{T}}=p P_{b}^{\mathrm{I}}+(1-p) P_{b}$, the total BER expression $P_{b}^{\mathrm{T}}$ for DCSK is obtained

$$
\begin{aligned}
P_{b}^{\mathrm{T}} & =\frac{p}{2} \operatorname{erfc}\left(\left(\frac{2\left(N_{0}+N_{I}\right)}{\sum_{l=1}^{L}\left|\alpha_{l}^{2}\right| E_{b}}+\frac{4 \beta\left(N_{0}^{2}+N_{I}^{2}\right)}{\left(\sum_{l=1}^{L}\left|\alpha_{l}^{2}\right| E_{b}\right)^{2}}\right)^{-0.5}\right) \\
& +\frac{1-p}{2} \operatorname{erfc}\left(\left(\frac{2 N_{0}}{\sum_{l=1}^{L}\left|\alpha_{l}^{2}\right| E_{b}}+\frac{4 \beta N_{0}^{2}}{\left(\sum_{l=1}^{L}\left|\alpha_{l}^{2}\right| E_{b}\right)^{2}}\right)^{-0.5}\right) .
\end{aligned}
$$

where $p=T_{n} \lambda$ is the impulsive noise occurrence probability.

\section{Discussions and Simulation Results}

In order to validate the accuracy of the total BER expression $P_{b}^{\mathrm{T}}$ obtained in the previous section and to compare the
TABLE I: Profile of the PLC multipath channel

\begin{tabular}{c|c|c}
\hline Path ID & Channel gains & delay $\left(\tau\left(T_{c}\right)\right)$ \\
\hline 1 & $0.3645-0.4860 i$ & 0 \\
2 & $0.3037+0.4252 i$ & 2 \\
3 & $0.1822-0.3645 i$ & 5 \\
4 & $0.3645-0.2430 i$ & 1
\end{tabular}

performance of DCSK to DS-CDMA and DS-DPSK systems, a simulation model was developed. Table I lists the channel parameters used in the simulation where the channel gains are normalized, such that $\sum_{l=1}^{L}\left|\alpha_{l}^{2}\right|=1$. Fig. 3a shows the BER of a DCSK modulator transmitting over a multipath channel, with and without the phase and impulsive noise, for $\beta=50$ and $E_{b} / N_{I}=7 \mathrm{~dB}$. Simulation results perfectly match the analytical expression derived for $P_{b}^{\mathrm{T}}$ for different values of impulsive noise occurrence probability $p$. Despite having a simple receiver design, DCSK is able to neutralize the effect of phase noise and compensate for the channel impairments. Note that a floor BER is observed for any $0<p \leq 1$. This is due to the superposition of both interference and impulsive noise. In the next experiment, the performance of DCSK is compared to coherent and non-coherent spread spectrum systems. To that end, the conventional DS-CDMA and DSDPSK systems are simulated. All three modulators transmit an identical data sequence over the same channel, with the characteristics described in Table I. For DS-CDMA and DSDPSK transmissions, Gold sequences are used to spread the data. The DS-CDMA receiver is equipped with a channel estimator that estimates the channel gain as well as the phase noise $\phi$. This channel estimation is erroneous due to the presence of noise in the PL channel. We plot the performance of the DS-CDMA system with and without a RAKE receiver. When the RAKE receiver is used, the system exploits the diversity gained due to multipath, but at the price of higher receiver complexity. On the other hand, the DCSK system is equipped with a much simpler receiver exhibiting high robustness to multipath and phase noise. In order to have a fair comparison between these three systems, the DS-DPSK receiver does not use a RAKE receiver. In this probe, we set the impulsive noise occurrence probability to $p=0.05$ and we maintain the variance of the phase noise as unity, i.e. $N_{0, \mu}=1$, for the duration of four symbols. Since the DSCDMA estimator is not perfect, the estimations of the channel gains and phase noise at the DS-CDMA receiver are done with error. Such an error is modelled as

$$
\begin{aligned}
& \hat{h}(t)=\sqrt{\rho} \sum_{l=1}^{L} \alpha_{l}+\sqrt{(1-\rho)} \varepsilon(t) \\
& \hat{\phi}(t)=\sqrt{\rho} \phi(t)+\sqrt{(1-\rho)} \varepsilon(t),
\end{aligned}
$$

where $0 \leq \rho \leq 1$ is the correlation factor and $\varepsilon(t)$ is a Gaussian random variable with zero-mean and unit variance representing the estimation error. When $\rho=1$, the DS-CDMA receiver estimates the channel and the phase noise parameters perfectly. When $\rho=0$, the receiver totally fails to correctly estimate the above quantities. The results of this experiment are shown in Fig. 3b. As expected, DS-CDMA with perfect channel estimation, i.e. $\rho=1$, has a superior performance over DCSK. This is owing to the fact the DS-CDMA is 


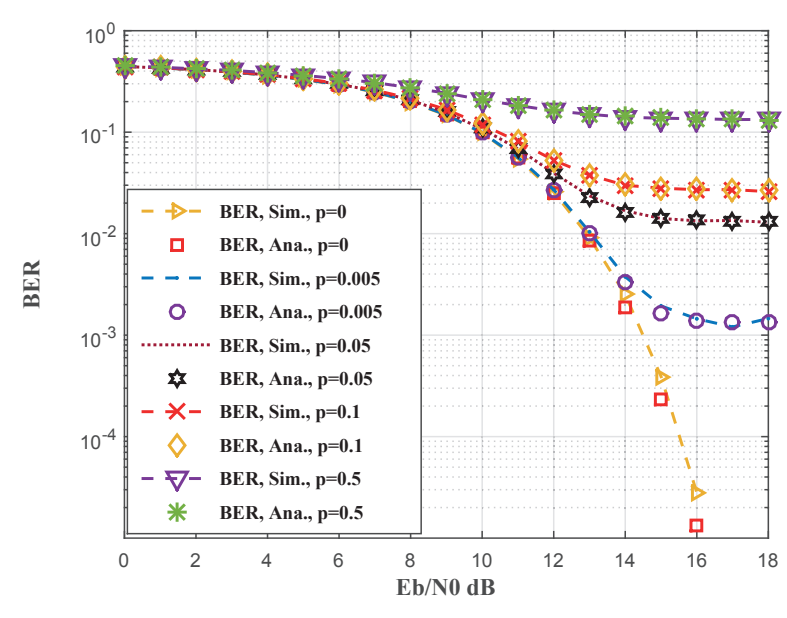

(a) Simulation vs. Analytical: $\operatorname{DCSK}(\beta=50)$.

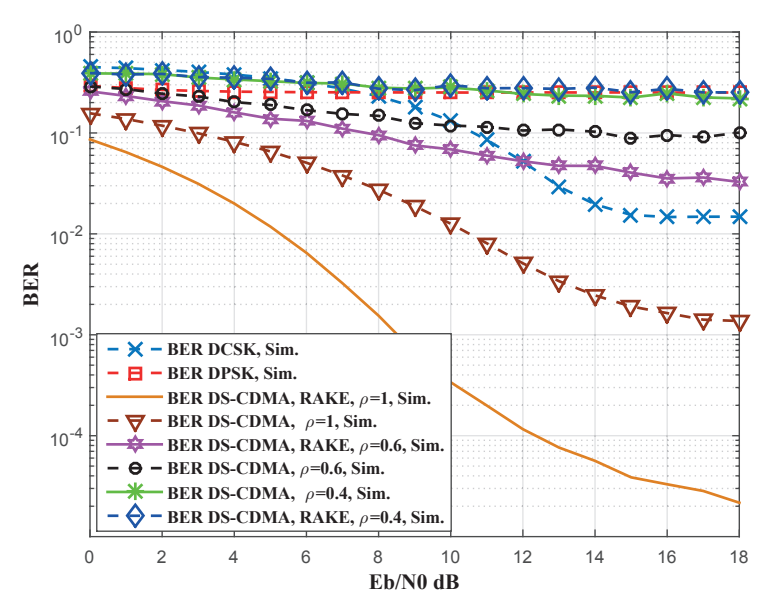

(b) Simulation: DCSK vs. DS-DPSK and DS-CDMA ( $\beta=63$, $p=0.05)$.

Fig. 3: Performance evaluation of DCSK system over multipath PLC channels.

equipped with a more complicated coherent receiver. In reality, the complexity in the structure of coherent CDMA receivers is the price that should be paid for a boost in performance. Since one of the prime objectives of the PLC paradigm is to have inexpensive receivers with robust performance, the expensive and complex DS-CDMA fails to stand out as a suitable choice. Nevertheless, as observed in Fig. 3b, DCSK offers a better performance in comparison to DS-CDMA for $\rho=0.4$ and $\rho=0.6$. On the other hand, DPSK shows a very weak resistance against phase noise and multipath interference. In fact, while DCSK offers a similar performance until $E_{b} / N_{0}=7 \mathrm{~dB}$, it outperforms DPSK for higher $E_{b} / N_{0}$ values. Based on the obtained results, we believe that DCSK can be suitable for PLC applications.

\section{CONCLUSIONS}

In this paper we propose a DCSK system as potential candidate for power-line communication (PLC) applications. The performance of DCSK system was analysed over power line channel with multiple echoes and in the presence of background, impulsive and phase noise. A closed-form bit error rate (BER) expression was derived and computer simulations were carried out to confirm the accuracy of this analytical finding. Our results indicate that the proposed system outperforms DSDPSK. When the channel estimation is erroneous, DCSK is superior to DS-CDMA too. For a perfect channel estimation, DS-CDMA provides a better performance at the expense of more complicated receiver design. Motivated by these facts, the DCSK system is introduced as a potential low-cost, robust modulation scheme for future PLC applications.

\section{REFERENCES}

[1] A. Sarafi, G. Tsiropoulos, and P. Cottis, "Hybrid wireless-broadband over power lines: A promising broadband solution in rural areas," IEEE Commun. Mag., vol. 47, no. 11, pp. 140-147, Nov. 2009.

[2] H. Philipps, "Modelling of Powerline Communication Channels," in IEEE International Symposium on Power Line Communications and Its Applications ISPLC, Mar. 1999, pp. 14-21.
[3] Y. Ma, P. So, and E. Gunawan, "Comparison of CDMA and OFDM systems for broadband power line communications," IEEE Trans. Power Del., vol. 23, no. 4, pp. 1876-1885, Oct. 2008.

[4] M. Zimmermann and K. Dostert, "Analysis and modeling of impulsive noise in broad-band powerline communications," IEEE Trans. Electromagn. Compat., vol. 44, no. 1, pp. 249-258, Feb. 2002.

[5] A. Mathur and M. R. Bhatnagar, "PLC Performance Analysis Assuming BPSK Modulation Over Nakagami-m Additive Noise," IEEE Commun. Lett., vol. 18, no. 6, pp. 909-912, Jun. 2014.

[6] A. Mathur, M. R. Bhatnagar, and B. K. Panigrahi, "PLC Performance Analysis Over Rayleigh Fading Channel Under Nakagami-m Additive Noise," IEEE Commun. Lett., vol. 18, no. 12, pp. 2101-2104, Dec. 2014.

[7] A. Mathur, M. R. Bhatnagar, and B. K. Panigrahi, "Performance Evaluation of PLC Under the Combined Effect of Background and Impulsive Noises," IEEE Commun. Lett., vol. 19, no. 7, pp. 1117-1120, Jul. 2015.

[8] R. Vali, S. Berber, and S. K. Nguang, "Analysis of Chaos-Based Code Tracking Using Chaotic Correlation Statistics," IEEE Trans. Circuits Syst. I: Reg. Papers, vol. 59, no. 4, pp. 796-805, Apr. 2012.

[9] G. Kaddoum, "Design and Performance Analysis of a Multiuser OFDM Based Differential Chaos Shift Keying Communication System," IEEE Trans. Commun., vol. 64, pp. 249-260, Jan. 2016.

[10] P. Chen, L. Wang, and F. Lau, "One Analog STBC-DCSK transmission scheme not requiring channel state information," IEEE Trans. Circuits Syst., vol. 60, no. 4, pp. 1027-1037, Mar. 2013.

[11] A. P. Kurian, S. Puthusserypady, and S. M. Htut, "Performance Enhancement of DS-CDMA System Using Chaotic Complex Spreading Sequence," IEEE Trans. Wireless Commun., vol. 4, no. 3, pp. 984-989, May 2005.

[12] T. S. Rappaport, Wireless Communications: Principles and Practice. Englewook Cliffs, NJ: Prentice-Hall, 1996.

[13] Y. Xia, C. K. Tse, and F. Lau, "Performance of differential chaosshiftkeying digital communication systems over a multipath fading channel with delay spread," IEEE Trans. Circuits Syst. II, Exp. Briefs, vol. 51, pp. 680-684, Dec. 2004.

[14] M. Zimmermann and K. Dostert, "A multipath model for the powerline channel," IEEE Trans. Commun., vol. 50, no. 4, pp. 553-559, Apr. 2002.

[15] M. Ghosh, "Analysis of the effect of impulse noise on multicarrier and single carrier QAM systems," IEEE Trans. Commun., vol. 44, no. 2, pp. 145-147, Feb. 1996.

[16] O. Hooijen, "On the channel capacity of the residential power circuit used as a digital communications medium," IEEE Commun. Lett., vol. 2, no. 10, pp. 267-268, Oct. 1998.

[17] E. Costa and S. Pupolin, "M-QAM-OFDM system performance in the presence of a nonlinear amplifier and phase noise," IEEE Trans. Commun., vol. 50, no. 3, pp. 462-472, Mar. 2002

[18] G. Kaddoum, P. Chargé, D. Roviras, and D. Fournier-Prunaret, "A Methodology For Bit Error Rate Prediction in Chaos-Based Communication Systems," Birkhäuser, Circuits, Systems and Signal Processing, vol. 28, pp. 925-944, Aug. 2009. 